\title{
A breath of fresh air
}

SARS-CoV-2: characterisation and mitigation of risks associated with aerosol generating procedures in dental practices.

BrDent J 2021; 230: https://doi.org/10.1038/s41415-020-2504-8.

Of all the new phrases that were adopted into the COVID-19 lexicon during 2020, 'fallow time' does characterise the unproductive frustration felt by many. Therefore, further research that can inform an evidence base to safely reduce fallow time and maximise productivity would be a welcome good news story.

To assess the best strategies to reduce potentially pathogen-carrying droplets that are aerosolised during dental procedures requires a degree of ingenuity and some fancy technology. The methodology in this paper involves some flexible tubes sucking air from around the dental surgery during a 'representative' aerosol generating procedure (AGP) and, in turn, funnelling the air into a 'high resolution electrical low-pressure impactor particle sizer'. It is made in Finland and is the type of technology used in car exhaust testing; it gives real-time measurements of particle number, size and concentration. The authors then compared various interventions against a control group of low-volume intraoral suction alone.

Using the proxy of the known diameter of an infectious coronavirus particle $(0.05-0.15 \mu \mathrm{m})$ as one of a range of measurements, the experiment assesses the effectiveness of various combinations of suction and an air cleaning system (ACS). The study showed that aerosol-management interventions substantially reduce, but do not eliminate, these particles during the AGP. The authors make it clear that none of these interventions are attempting to replace strict PPE protocols for practitioners during the procedure.

The ACS in this case was a high-pressure air purifier that increases in-surgery air changes per hour to 24 . The key being not just the increased filtration, but an electrostatic plasma function that can eliminate smaller particles that evade a filter, such as virus particles.

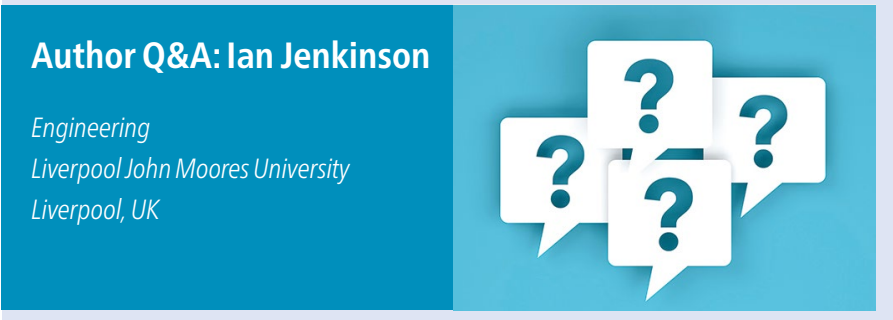

Why did you decide to undertake this study?

Dental clinics were closed for a period of approximately three months because of the COVID pandemic. Since reopening, practices have experienced a substantial loss in productivity due to the implementation of a 'fallow time' between patients. This study was initiated to better understand dental aerosol generating procedures (AGPs), so that dental hospitals, practices, labs and associated dental supply chain businesses can remain open and operate safely through any future viral pandemics.

\section{Did any of the results surprise you?}

Yes. The particle size distribution in the aerosols generated from AGPs were predominantly $<0.3 \mu \mathrm{m}$ in diameter. This encompasses the reported size range of the SARS-CoV-2 virus $(0.05-0.15 \mu \mathrm{m})$. In the absence of any

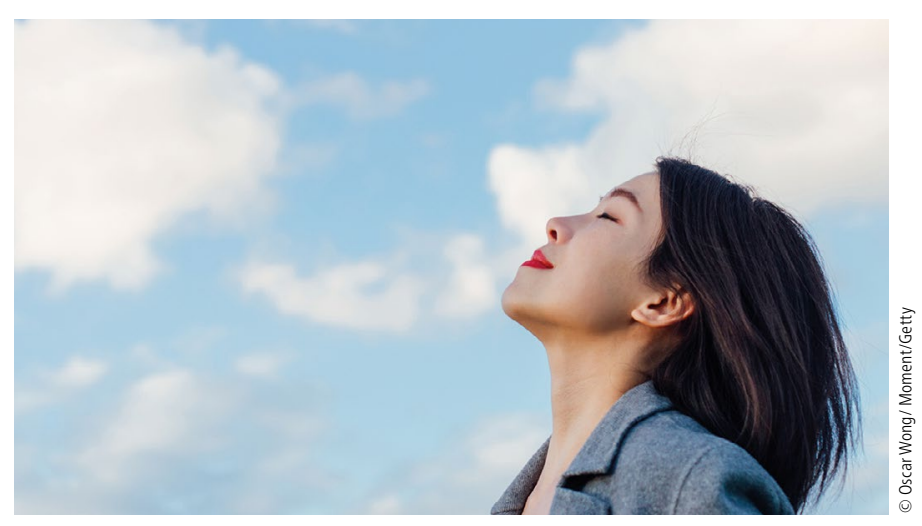

After some number crunching, the combination of intraoral lowvolume and high-volume suction, and an ACS are suggested to reduce fallow time below ten minutes, even eliminate it altogether. Interestingly, that addition of high-volume extraoral suction might not be helpful.

Limitations, as set out by the authors of the study, are the use of a phantom head which may not be an exact replacement for a living, salivating, spluttering patient. The specifics of the environment need to be considered as well; in this case an average-sized dental surgery is probably comparable to most clinical environments, with the exception of open-plan clinics in a dental hospital.

All of this hints at an end to fallow time, which would be a breath of fresh air.

By Sam McCarthy

Speciality Doctor in Oral Medicine, Newcastle Dental Hospital

active aerosol-management interventions, residual AGP particles were detected for up to 30 minutes after completion of the AGPs.

Even in the presence of interventions such as intra-oral low and high-volume suction (L/HVS[IO]), either alone, or combined with an air cleaning system (ACS), aerosol particles $<0.3 \mu \mathrm{m}$ were substantially elevated above the baseline range, and significantly within the space of $50 \mathrm{~cm}$ of the patient's mouth. This emphasised the importance of properly fitted personal protective equipment such as FFP3 masks.

\section{What do you think the next steps should be considering your findings?} L/HVS(IO) combined with the ACS eliminated fallow time and controlled the dose of aerosol particles in the surgery. The ACS delivered 24 air changes per hour in a $35 \mathrm{~m}^{3}$ surgery, which was close to maximum. Further experimental work is needed to optimise the location and setting of the equipment. The efficacy of HVS IO compared to HVS EO also needs to be assessed, as HVS IO can be susceptible to set-up and operator technique.

A patient-focused study is needed to confirm the nature of the fine aerosols which will contain a mixture of saliva, coolant, and pathogens. A study of the effectiveness of FFP3 face masks with such small aerosol size ranges is also needed. 